\title{
DCM: D Number Extended Cognitive Map. Application on Location Selection in SCM
}

\author{
L. Zhao, F. Xiao
}

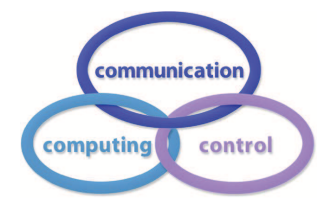

\author{
Lian Zhou \\ Computer and Information Science \\ Southwest University, Chongqing, China \\ Fuyuan Xiao* \\ Computer and Information Science \\ Southwest University, Chongqing, China \\ *Corresponding author: xiaofuyuan@swu.edu.cn, doctorxiaofy@hotmail.com
}

\begin{abstract}
Offshore outsourcing is a widely used management technique for performing business functions with the aim of reducing labor and transportation costs. The selection of locations has a significant influence on the supply chain's resilience and qualities, but the influence of multiple external factors on the supply chain's performance in local places in a complex and uncertain environment has not been examined. In this study, we investigated the influence of external factors in a highly uncertain and complicated situation in which relationships between external factors and supply chain resilience are complicated. Furthermore, we proposed a novel model to select locations from a comprehensive perspective. Specifically, the fuzzy cognitive map (FCM) is utilized to simulate the dynamic influence process where the adjacency is aggregated by D numbers. The weights of different resilience capabilities are considered from the perspective of maximizing benefits by using the decision-making trial and evaluation laboratory-analytic network processes (DEMATEL-ANP) model. By comparing the distance to the ideal solutions, we selected the best alternative location. Our results differ from the general case, which reveals that the weights of different capabilities influence selections.

Keywords: Offshore outsourcing, supply chain resilience, location selection; FCM, D number, DEMATEL-ANP, multicriteria decision making (MDM).
\end{abstract}

\section{Introduction}

In recent years, the globalization of the economy and competition has led to low consumption, and thus, the offshore outsourcing process has attracted extensive attention as one of the most adaptive strategies $[9,13]$. Offshoring allows for firms to focus on core competencies to improve their productivity, efficiency, and flexibility. The core competencies release a range of expertise that crosses traditional functions horizontally and adapts to changes in the long-term demands of clients, thus continuing domination over competitors [21]. A comparatively successful offshore outsourcing strategy can significantly assist a firm in improving its production efficiency, productivity, and flexibility to improve total profits and tackle emergency situations [22]. However, factors limiting the extensive application of offshore outsourcing remain, and outsourcing may elicit some potential threats to client firms by extending their supply chains (SCs) [6].

Offshore outsourcing also has the potential to transfer specific ownership of the business 
activities and resources to low-cost providers overseas. Comparing the keyword offshore outsourcing to a similar keyword, outsourcing, we usually consider offshore outsourcing to involve a vendor located in a country other than that of the buyer. Therefore, only the location of the seller differentiates the above two keywords. The core of offshore outsourcing is the practice of using a supplier rather than in-house employees to perform a function [2]. The following question to be solved is where to outsource [7]. The selected provider will participate in increasing integration of the SC. Previous research has shown that the supply chain network is vulnerable to disruptions, and the failure of elements within the SC may cause the failure of the whole network. We assumed that an ideal offshore outsourcing location would satisfy the requirements of employers in terms of both economic and political aspects to enhance the performance of the whole SC. However, an erroneous choice may cause a significant negative effect on the entire SC network [20].

To realize competitive advantages of supply chains, the priority is to develop 'agility', 'selfalignment', and 'adaptation' (triple A's). Supply chain 'agility' indicates an instant response to short-term perturbations caused by uncertainties in the upstream and downstream supply chains. With respect to offshore outsourcing supply chains, agility is related to the capabilities of the supply chain to deal with unexpected changes in market demand, which can transform from challenges into opportunities and lead the supply chain to gain competitive advantages in a volatile and turbulent environment [33]. Supply chain 'alignment' refers to the integration process of several members in the supply chain to achieve better performance [25]. The necessity of the attribute has been highlighted in many studies and requires further investigation [35]. Finally, the adaptability of the supply chain enables the supply chain to evolve based on market changes in strategies [15]. Recent researches have shown the importance of supply chain adaptability as a dynamic capability. In summary, the reviewed capabilities could be denoted by the keyword 'resilience', and more specifically, the best way to improve a supply chain's competitiveness in an offshore outsourcing scenario is to maximize its resilience.

Most client firms are offshoring core competencies with global suppliers, with a focus on the selection of key value-creating competencies. This situation increases SC complexity and interdependency, leading to a risk of SC vulnerability. Therefore, SC resilience is very crucial to minimize property losses in disruptions. Recent research has revealed that the Tohoku earthquake affected supply chains in the Philippines in 2017. Specifically, due to the lack of resilience in supply chains, many properties and client firms suffered from destructive natural disasters [1].The above event suggests that a resilient supply chain is important for minimizing the negative effects of disruptions and allowing for the $\mathrm{SC}$ to recover normal activity in a short time [9].If a corporation has a responsive SC, SC resilience can return to a normal or even better state [10]. Furthermore, an offshore outsourcing process also involves unexpected outer disruptions and accidental events, and incoming potential risks would cause damage to SC systems. Moreover, due to the complexity and interdependence of entities in SC, a minor failure within the SC may cause a failure cascade in the whole system. Therefore, SC resilience is considered an important indicator in the offshore outsourcing process.

Despite these challenges, previous research has not made progress in investigating the relationship between locational decisions in offshore outsourcing and SC resilience. Few studies have employed SC resilience as an indicator when selecting offshore outsourcing locations. Thus, we assumed that an ideal offshore outsourcing location has positive effects on SC resilience to prevent accidental disruptions and ensure SC robustness. To bridge the gap between offshore outsourcing and SC resilience, this study proposed a combined method based on the analytic network process (ANP) [12], decision-making trial and evaluation laboratory (DEMATEL) method [11], fuzzy cognitive map (FCM) [12], and technique for order preference by similarity to an ideal solution (TOPSIS) [16]. Specifically, FCM can represent all connections with the considered 
abilities to evaluate alternative locations and address the imprecise and fuzzy weights of links. DEMATEL and ANP are combined to determine the weights of FCM, and TOPSIS is implemented in alternative locations specific to outcomes gathered from FCM.

In this study, FCM can predict the impacts of alternative locations on SC resilience by simulating scenarios over time, and the executive functions can select an optimal offshore outsourcing location. Furthermore, ANP-DEMATEL is used to determine the weights of links by inputting evaluations from experts. In addition, TOPSIS is considered an efficient tool for ranking alternative offshore outsourcing locations with respect to SC resilience. The aim is to maximize SC resilience by choosing an ideal offshore outsourcing location. In addition, to avoid subjectivity and fuzziness of experts assessments, multiple experts are invited to evaluate the project, and their opinions are aggregated by D numbers The rest of this paper is organized as follows. The background of our study is overviewed in Section 2. In Section 3, some related preliminaries related to our study are introduced. In Section 4, we present our hybrid integration approach for selecting the best alternative outsourcing locations with respect to supply chain resilience.we present a DCM based integration decision model for selecting the best alternative outsourcing locations with respect to supply chain resilience. Section 5 presents an empirical application of the proposed model. The verification and discussion of our approach's rationality and superiority are described in Section 6. Section 7 conducts a sensitivity analysis. In the final section, we present the conclusions of our study.

\section{Preliminaries}

\section{$2.1 \quad$ D number theory}

In the real world, uncertainty modeling and optimization are difficult to quantify because knowledge and information are imprecise and incomplete [14]. Dempster-Shafer evidence theory (also known as D-S theory or evidence theory) is an efficient tool for dealing with the information fusion issue $[3,4,17,28]$. However, some inherent drawbacks limit the broader application of the D-S theory. First, D-S theory requires a strong hypothesis that elements in the discerning frame are mutually exclusive, which is very difficult to satisfy, especially in linguistic assessments. Second, a norm basic probability assignment (BPA) must follow a completeness constraint in D-S theory, which means that the sum of all focal elements in a BPA must be equal to 1 [6]. However, the experts do not have access to the overall knowledge, and the assessment is based only on partial information, potentially resulting in an incomplete BPA $[32,33]$. To address this issue, D numbers are presented [8,18-20,29].

\subsection{Fuzzy cognitive maps}

Political scientist Robert Axelrod introduced cognitive maps in the 1970s to represent social scientific knowledge [23]. The fuzzy cognitive map (FCM), an extension of the cognitive map, is a causal description of a model of the behavior of a system [24]. FCM is an interactive structure of concepts, each of which interacts with the rest, and reveals the dynamics and different behaviors of the system. Each concept is described by a number $A_{i}$ that represents its value and results from the transformation of the fuzzy real value of the system's variable, for which this concept stands, in the interval $[0,1]$. There are three types of causal interactions between concepts that represent the type of influence from concepts to the others. 


\subsection{DEMATEL}

The methodology of the Decision Making Trial and Evaluation Laboratory (DEMATEL) was developed initially by the Battelle Memorial Association in Geneva [5] and is an effective method for analyzing the direct and indirect relationships between components in the system with respect to severity and type [34]. DEMATEL is widely applied in supply chain management and service quality evaluation. By utilizing this method, we can extract a better understanding of the structural relationships, and thus, this method is an ideal way to solve complicated system problems. The procedure for implementing DEMATEL to solve dependent evidence consists of four steps namely 1) Define the quality feature and establish the measurement scale 2) Extract the DRM of influential factors 3) Normalized DRM 4) Calculate TRM.

\subsection{ANP}

Network analysis is a very useful tool to model real application [31,36,37]. The analytic network process (ANP) is capable of tackling dependence among components in a system. It is a generation of the analytic hierarchy process (AHP) and allows for more complicated interrelationships among decision elements by replacing the hierarchy in the AHP [26] with a network. The network structure of ANP includes the control level and network level. The control level consists of goal and independent criteria whose weights can be obtained by AHP. There is at least one goal at the control level. At the network control level, the network spreads out in all directions and involves arrows between clusters or loops within the same cluster. These arrows and loops indicate the relations among clusters or within a cluster. ANP is also applied to prioritize factors or criteria in the decision-making problem. In this paper, we only consider one goal, and criteria are omitted at the control level.

\subsection{TOPSIS}

The technique for Order Preference by Similarity to Ideal Solution (TOPSIS), which was developed by Hwang and Yoon in 1981 [38], is a method for ranking alternatives in applications and concepts $[30,39]$. The core ideal of TOPSIS is to choose alternatives that simultaneously have the shortest distance from the positive ideal solution and the farthest distance from the negative ideal solution. The positive ideal solution maximizes the benefit criteria and minimizes the cost criteria, whereas the negative ideal solution maximizes the cost criteria and minimizes the benefit criteria.

\section{Selecting offshore outsourcing location considering supply chain resilience}

As shown above, supply chain resilience (SCRE) is a crucial factor in offshore outsourcing and should receive close attention when selecting an outsourcing location. The main thrust of this paper is to rank some alternative locations and select the most suitable one from the perspective of SCRE. However, in the real world, multiple factors influence SCRE, and these factors interact with SCRE under changing conditions in foreign locations. Thus, the interacting behaviors among SCRE and these external factors should be regarded as a dynamic mechanism in a highly complex and uncertain manner. In addition, the SCRE could be divided into various capabilities (e.g., flexibility, visibility, anticipation, etc.). These different capabilities have weights corresponding to different criteria under one goal of maximizing total benefits. All of these issues should be taken into consideration via a comprehensive perspective. In summary, the issue 
of selecting the best offshore outsourcing location is a typical multi-criteria decision-making (MCDM) problem. Since most existing research has researches have regarded the issue as a static problem or focused on one aspect of it, we propose a comprehensive perspective to tackle this complicated problem. First, we use a fuzzy cognitive map extended by the D number to identify the influencing mechanism of outer factors on SCRE in an offshore outsourcing location. Then, an ANP-DEMATEL model is utilized to assign weights to different capabilities of SCRE in the benefit perspective. Finally, we rank alternative locations with disparate outer factors by using the TOPSIS method. The details are introduced below.

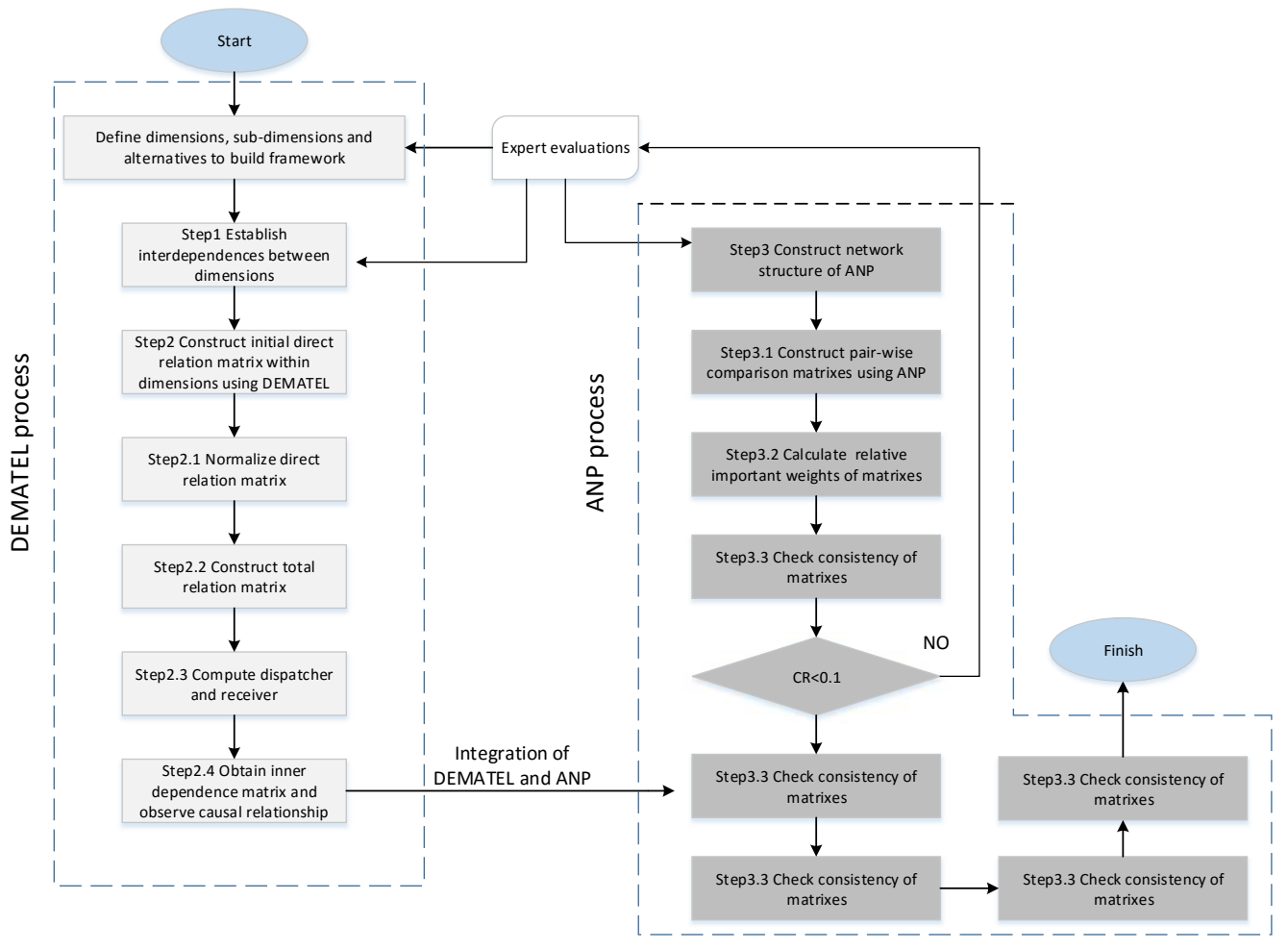

Figure 1: An overview of the weight assignment framework

\subsection{Identify the impacts of outer factors on SCRE}

To determine the impact of outer factors on SCRE in offshore outsourcing, the FCM is introduced to simulate the process in a complicated and uncertain environment. The procedure is presented below.

1) Expert evaluation: Some authorities on outsourcing will be invited to define crucial outer factors that are closely related to SCRE. They may use their experience, knowledge and some interactive techniques such as the Delphi method or affinity diagrams to identify these factors for further research. Similarly, the different aspects of SCRE capability are addressed in this step. These outer factors and the SCRE capabilities will be regarded as the nodes in the FCM that describe the real problem or general system.

2) Identify the causal connections between nodes $\left(w_{i} \rightarrow w_{j}\right)$. The type of relation (positive or negative) and its intensity should be taken into consideration and defined. The preliminary means of conducting this procedure is to define intensity scales. All evaluations should be mapped on the interval $[0,1]$. We use AHP to quantify the degree of intensity. The scientific evaluations 
are transformed into a quantitative range by implementing the AHP method.

3) Aggregate the multiple experts' opinions in different groups. In the real world, the interactions between external factors and SCRE capabilities are very uncertain due to their complexity. To address this problem, multiple experts with different backgrounds will be invited to decrease uncertainty and obtain a relatively precise result. D number theory is introduced to fuse these evaluations due to its superiority in representing incomplete and imprecise information.

4) FCM inference. FCM can not only represent causal relations but also predict future implications through dynamic simulations. At the initial stage, each FCM node is assigned a value $[0,1]$, and the initial state vector $V_{s_{i}}^{0}=\left(\begin{array}{lllll}v_{1}^{0} & v_{2}^{0} & \cdots & v_{n-1}^{0} & v_{n}^{0}\end{array}\right)$ is obtained. Then, using Eq. (7), the behavior of the influencing mechanism will be revealed.

In this stage, the impacts of external factors on SCRE can be identified, and the evolutionary process of FCM can quantify these impacts.

\subsection{Weight different resilience capability}

An overview of the integrated DEMATEL and ANP approach for assigning different weights to SCRE capabilities is given in Fig. 1.

Briefly, the values are gathered from a computer database and questionnaires. The DEMATEL method is employed to deal with the inner dependency by gathering pairwise comparisons. The inner dependency is then structured, and ANP is implemented to perform further calculations. The details are listed as follows:

Step 1: Define the criteria, objective, sub-criteria, and alternatives of the ANP model. The criteria and sub-criteria are acquired from experts' knowledge, experience, and other appropriate approaches. In this paper, the objective is to maximize the offshore outsourcing practitioners' benefits, and the alternatives are different SCRE capabilities.

Step 2: Construct a direct-relation matrix using DEMATEL: Decision makers are required to compare the criteria pairwise with respect to influence and direction. The comparison scale is defined at 5 levels: (0) no influence, (1) low influence, (2) medium influence, (3) high influence, and (4) extreme influence.

Step 2.1: Normalize the direct-relation matrix. The direct-relation matrix is transformed to the normalized matrix.

Step 2.2: Calculate the total relation matrix. The total relation matrix could be obtained via the normalized direct-relation matrix.

Step 2.3: To visualize the relations among factors, we calculate the dispatcher and receiver groups. The dispatcher is estimated from $D-R$, which has positive values and a greater influence on other factors. They are assumed to exhibit higher priority and are called dispatcher groups, where $\mathrm{R}$ is the sum of the columns and $\mathrm{D}$ is the sum of rows in the matrix $\mathrm{T}$. The other values with negative values of $D-R$ receive more influence from one another and are considered to have lower priority; these are called receiver groupers. The value $D+R$ here shows the relation degree of each factor with others. Those factors with higher $D+R$ have more of a relationship with each other, and those with lower $D+R$ have less of a relationship with each other.

Step 2.4: Obtain the inner dependence matrix: The sum of each column is 1 in the total relationship matrix.

Step 3: Use ANP to construct the network for the problem and access the different SCRE capabilities.

Step 3.1: Calculate the relative weights of different criteria in offshore outsourcing benefits. The AHP method is utilized to calculate their weight. A scale of 9 from equal importance to extreme importance is used to measure the relative importance of criteria. A decision maker would judge the relative dominance between each pair of criteria, and the results will be turned 
into values of $1,3,5,7$, and 9 , which represent equal importance, moderate importance, strong importance, demonstrated importance, and extreme importance, respectively, whereas values of $2,4,6$, and 8 indicate intermediate importance. Through the process of AHP, the relative weights of criteria are obtained.

Step 3.2: The supermatrix can be acquired by entering the vectors derived from DEMATEL, and then, the normalized weighted matrix is transferred from the supermatrix by multiplying the relative weights of criteria from step 3.1., in which every column's sum is 1 . The weighted matrix then converges to a stable value after it is raised by its limiting power.

Step 4 Determine the relative weights of the SCRE capabilities: the final weights are found in the corresponding row in the limit of the supermatrix; these weights should be taken into consideration when we select optimal offshore outsourcing locations.

\subsection{Rank alternative locations and select optimal one in SCRE perspective}

Some alternative locations for offshore outsourcing are discussed and compared at this stage. Since these alternatives have different outer factors, they have different values for each outer factor. We input these initial vectors into the FCM, and after the FCM reaches an equilibrium stage, various SCRE capabilities will be activated. The TOPSIS method is then used to quantify the distance of alternative locations to the ideal solution considering the relative weights of each SCRE capability. The location with the greatest value according to Eq. (18) will be chosen as the suitable location for offshore outsourcing.

\section{Empirical case study}

In this section, we present an empirical case to illustrate the effectiveness of our method for location selection considering SCRE. Four alternative locations are considered in our study. This study pursues a generalized finding and proves the proposed method's effectiveness. In this study, we have been required to select the best offshore outsourcing location considering SCRE capability in the background of a single and explanatory case study. The chosen case is highly representative and valuable because it evaluates the global supply chain of spirit drinks with geographically remote locations worldwide. The supply chain is so complicated that only the focal producer has more than 30 production facilities. We will use the proposed method to select the most suitable locations step by step.

\subsection{Identify the impacts of outer factors on RSCRE}

1) Expert evaluation: Some experts are invited to describe outer factors that influence the resilience of the supply chain as well as the detailed capabilities of SCRE. The results are shown in the Appendix. More specifically, the nodes in the FCM will be acquired.

2) In this step, the experts judge the causal relations among the nodes as well as their intensity. AHP is implemented to make pairwise comparisons, and quantitative intensities are obtained. All experts have the same weights; thus, the outcome will be the average of all experts. The consistency ratio should be less than 0.1 , and all numbers map into $[0,1]$. The intensity levels are shown in Table 1.

3) Aggregate experts' opinions from different groups. Since the causal relations among nodes are highly complicated and uncertain in practice, multiple experts are invited to assess the causal relations among those external factors and capabilities. In this case, three groups of experts are invited to evaluate the causal relations between them. These three groups have different backgrounds, and they will give their opinions from different perspectives. The impact 
Table 1: Intensity levels and their associated quantities from AHP

\begin{tabular}{lllll}
\hline Intensity level & Quantity(expert1) & Quantity(expert2) & Quantity(expert3) & Average \\
\hline None & 0 & 0 & 0 & 0 \\
Very Weak & 0.03 & 0.055 & 0.026 & 0.037 \\
Weak & 0.06 & 0.062 & 0.076 & 0.066 \\
Moderate & 0.117 & 0.143 & 0.121 & 0.127 \\
Strong & 0.225 & 0.264 & 0.256 & 0.248 \\
Very Strong & 0.568 & 0.477 & 0.521 & 0.522 \\
Consistency Ratio & 0.07 & 0.07 & 0.07 & 0.045 \\
\hline
\end{tabular}

of F12 (technological infrastructure) on Cap9 (collaboration) can be considered as an example. In the first group, seven experts conclude that technological infrastructure has a strong positive relationship with collaboration; two experts believe the relationship is solid, and only one thinks it is moderate. In the second group, six experts assign a strong relation between F12 and Cap9; one holds the view of a moderate relationship. And the results are shown in Table 2 and Table 3. See also in Appendix the interactions between the criteria and SC resilience capabilities.

Table 2: The adjacency matrix (Part A)

\begin{tabular}{|c|c|c|c|c|c|c|c|c|c|c|c|c|c|c|c|c|}
\hline ID & F1 & $\mathrm{F} 2$ & F3 & $\mathrm{F} 4$ & F5 & F6 & F7 & F8 & F9 & F10 & F11 & F12 & F13 & F14 & F15 & F16 \\
\hline F1 & 0 & 0 & 0 & 0 & 0 & 0 & 0 & 0 & 0 & 0 & 0 & 0 & 0 & 0 & 0 & 0 \\
\hline $\mathrm{F} 2$ & 0 & 0 & 0 & 0 & -0.533 & 0 & 0 & 0 & 0.251 & 0 & 0 & 0 & 0 & 0 & 0 & 0 \\
\hline F3 & 0 & 0 & 0 & 0 & 0 & 0 & 0 & 0 & 0 & 0 & 0 & 0 & 0 & 0.523 & 0 & 0.248 \\
\hline F4 & 0 & 0 & 0 & 0 & 0 & 0 & 0 & 0 & 0 & 0 & 0 & 0 & 0 & 0 & 0 & 0 \\
\hline F5 & 0 & 0 & 0 & 0 & 0 & 0 & 0 & 0 & 0 & 0 & 0 & 0 & 0 & 0 & 0 & 0 \\
\hline F6 & 0 & 0 & 0.254 & 0 & 0 & 0 & 0 & 0 & 0 & 0 & 0 & 0 & 0 & 0 & 0 & 0 \\
\hline F7 & 0 & 0 & 0 & 0 & 0 & 0 & 0 & 0 & 0 & 0 & 0 & 0 & 0 & 0 & 0 & 0 \\
\hline F8 & 0 & 0 & 0 & 0 & 0 & 0 & 0 & 0 & 0 & 0 & 0 & 0 & 0 & 0 & 0 & 0 \\
\hline F9 & 0 & 0 & 0 & 0 & 0 & 0 & 0 & 0.067 & 0 & 0.248 & 0 & 0 & 0 & 0.251 & 0 & 0 \\
\hline F10 & 0 & 0 & 0 & 0 & 0 & 0 & 0 & 0 & 0 & 0 & 0 & 0 & 0 & 0 & 0 & 0 \\
\hline F11 & 0 & 0 & 0 & 0 & 0 & 0 & 0 & 0 & 0 & 0 & 0 & 0 & 0 & 0 & 0 & 0 \\
\hline F12 & 0 & 0 & 0 & 0 & 0 & 0 & 0 & 0 & 0 & 0 & -0.067 & 0 & 0 & 0 & 0 & 0 \\
\hline F13 & 0 & 0 & 0 & 0 & 0 & 0 & 0 & 0 & 0 & 0.071 & 0 & 0 & 0 & 0.251 & 0 & 0 \\
\hline F14 & 0 & 0 & 0 & 0 & 0 & 0 & 0 & 0 & 0 & 0 & 0 & 0 & 0 & 0 & 0 & 0 \\
\hline F15 & 0 & 0 & 0 & 0 & 0 & 0 & 0 & 0 & 0 & 0 & -0.067 & 0 & 0 & 0 & 0 & 0 \\
\hline F16 & 0 & 0 & 0 & 0 & 0 & 0 & 0 & 0 & 0 & 0 & 0 & 0 & 0 & 0 & 0 & 0 \\
\hline Cap1 & 0 & 0 & 0 & 0 & 0 & 0 & 0 & 0 & 0 & 0 & 0 & 0 & 0 & 0 & 0 & 0 \\
\hline Cap2 & 0 & 0 & 0 & 0 & 0 & 0 & 0 & 0 & 0 & 0 & 0 & 0 & 0 & 0 & 0 & 0 \\
\hline Cap3 & 0 & 0 & 0 & 0 & 0 & 0 & 0 & 0 & 0 & 0 & 0 & 0 & 0 & 0 & 0 & 0 \\
\hline Cap4 & 0 & 0 & 0 & 0 & 0 & 0 & 0 & 0 & 0 & 0 & 0 & 0 & 0 & 0 & 0 & 0 \\
\hline Cap5 & 0 & 0 & 0 & 0 & 0 & 0 & 0 & 0 & 0 & 0 & 0 & 0 & 0 & 0 & 0 & 0 \\
\hline Cap6 & 0 & 0 & 0 & 0 & 0 & 0 & 0 & 0 & 0 & 0 & 0 & 0 & 0 & 0 & 0 & 0 \\
\hline Cap7 & 0 & 0 & 0 & 0 & 0 & 0 & 0 & 0 & 0 & 0 & 0 & 0 & 0 & 0 & 0 & 0 \\
\hline Cap8 & 0 & 0 & 0 & 0 & 0 & 0 & 0 & 0 & 0 & 0 & 0 & 0 & 0 & 0 & 0 & 0 \\
\hline Cap9 & 0 & 0 & 0 & 0 & 0 & 0 & 0 & 0 & 0 & 0 & 0 & 0 & 0 & 0 & 0 & 0 \\
\hline
\end{tabular}


Table 3: The adjacency matrix (Part B)

\begin{tabular}{cccccccccc}
\hline ID & Cap1 & Cap2 & Cap3 & Cap4 & Cap5 & Cap6 & Cap7 & Cap8 & Cap9 \\
\hline F1 & 0 & 0 & 0 & 0 & 0 & 0 & 0 & 0.531 & 0 \\
F2 & 0 & 0.251 & 0 & 0.133 & 0 & 0 & 0 & 0 & 0 \\
F3 & 0 & 0 & 0 & 0 & 0 & 0 & 0 & 0 & 0 \\
F4 & 0 & 0 & 0 & 0 & 0 & 0 & 0 & 0 & 0 \\
F5 & -0.127 & 0 & 0 & -0.253 & 0 & 0 & 0 & -0.131 & 0 \\
F6 & 0 & 0 & 0 & 0 & 0 & 0 & 0 & 0 & 0 \\
F7 & 0 & 0 & 0.252 & 0 & 0.526 & 0 & 0 & 0 & 0 \\
F8 & 0 & 0 & 0 & 0 & 0 & 0 & -0.071 & 0 & 0 \\
F9 & 0 & 0 & 0 & 0 & 0 & 0 & 0 & 0 & 0 \\
F10 & -0.388 & 0 & 0 & -0.251 & 0 & 0 & -0.131 & 0 & -0.04 \\
F11 & 0 & -0.066 & -0.127 & 0 & 0 & 0 & 0 & 0 & 0 \\
F12 & 0.248 & 0.248 & 0.248 & 0 & 0.129 & 0 & 0 & 0 & 0.279 \\
F13 & 0 & 0 & 0 & 0 & 0 & 0 & 0 & 0 & 0 \\
F14 & 0 & 0 & 0 & -0.068 & 0 & 0 & -0.131 & 0 & 0 \\
F15 & 0 & 0 & 0 & 0 & 0 & 0.068 & 0 & 0 & 0.131 \\
F16 & -0.525 & 0 & 0 & 0 & 0 & 0 & 0 & 0 & 0 \\
Cap1 & 0 & 0 & 0 & 0 & 0 & 0 & 0 & 0 & 0 \\
Cap2 & 0 & 0 & 0 & 0 & 0 & 0 & 0 & 0 & 0 \\
Cap3 & 0 & 0 & 0 & 0 & 0 & 0 & 0 & 0 & 0 \\
Cap4 & 0 & 0 & 0 & 0 & 0 & 0 & 0 & 0 & 0 \\
Cap5 & 0 & 0 & 0 & 0 & 0 & 0 & 0 & 0 & 0 \\
Cap6 & 0 & 0 & 0 & 0 & 0 & 0 & 0 & 0 & 0 \\
Cap7 & 0 & 0 & 0 & 0 & 0 & 0 & 0 & 0 & 0 \\
Cap8 & 0 & 0 & 0 & 0 & 0 & 0 & 0 & 0 & 0 \\
Cap9 & 0 & 0 & 0 & 0 & 0 & 0 & 0 & 0 & 0 \\
\hline
\end{tabular}

4) FCM inference. The FCM nodes' values are assigned to $[0,1]$ to simulate the impacts of outer factors on SCRE capabilities. In this study, we set the initial state vector $V_{s_{i}}^{0}=\left(\begin{array}{llllll}0 & 0 & \cdots & 1 & \cdots & 0\end{array}\right) \quad(n=1,2, \cdots, 16)$. The number in the initial state vector represents the external factors, and our one factor is given a value of 1 ; the outer factors are assigned to 0 . The activation value of the SCRE capabilities can be obtained. In this way, a better perspective of the impact of each outer factor on the SCRE capabilities is provided. In Appendix can be see the impact of each outer factor on the 9 SCRE capabilities and the influence of each outer factor on the SCRE capabilities. F12 (technological infrastructure) has the most positive effects on SCRE, whereas F9 (tax rate) significantly impairs the whole resilience of the supply chain. The conclusion drawn from the table is that the offshore outsourcing practitioner should select locations with great technical infrastructure and a lower tax rate.

\subsection{Weight different resilience capability}

Step 1: The objective, criteria, sub-criteria, and alternatives of the decision-making problem are introduced in Section 3.2. Finally, 5 criteria, as well as 21 of their sub-criteria, are identified:

Technical aspects: (C1) Efficiency, (C2) Security, (3) Energy sustainability, (4) Circulation of capital, (5) Advanced technology, and (6) Technical innovation

Economic aspects: (C7) Operation cost, (C8) Investment cost, (C9) Production cost, (C10) Return on investment, and (C11) Maintenance cost

Political aspects: (C12) Foreign dependency, (13) Capability with respect to the national political and legislative situation, (14) Capability with respect to national energy policy objectives, and (15) Public policy and financial support

Social aspects: (C16) Social benefits, (17) Social acceptability, and (18) Job creation

Environmental aspects: (C19) Greenhouse emissions, (20) Land requirements, and (21) Impact on the ecosystem

Step 2: Construct direct relation matrixes according to the 4-level scale, which features inner dependence. Taking $\mathrm{C} 7-\mathrm{C} 11$ as an example, the initial relations among them are listed in 
the Appendix. Not all direct relation matrixes are listed here due to space limitations.

Steps 2.1-2.2: The direct relation matrixes can be transformed into total relation matrixes to reveal their comprehensive relations. Still taking C7-C11 as an example, the overall relation matrix is shown in the Appendix.

Step 2.3: The impact diagram is constructed as introduced in Section 3. The horizontal axis refers to $\mathrm{R}+\mathrm{D}$, and the vertical axis is $\mathrm{D}-\mathrm{R}$. The impact diagram visualizes the direct and indirect relations, where $\mathrm{D}+\mathrm{R}$ is the sum of relations among the elements and shows the importance of each element. D-R indicates the causal relations; $D-R>0$ indicates that the element affects other elements to a greater extent than it is affected by them. The impact diagram of C7-C11 is shown in Appendix.

Step 2.4: The results reveal a strong inner dependence among the sub-criteria of the economic aspect. Thus, the inner dependence matrix is calculated. Table 8 in the appendix shows the inner dependence matrix of the economic aspect.

Taking $C 7-C 9$ as an example, the total relation of C7-C9 is 0.101 , and $R_{C 7}=0.101+0.243+$ $0.088+0.111+0.119=0.661$. Therefore, the inner dependence of $\mathrm{C} 7-\mathrm{C} 9$ is $0.101 / 0.661=0.152$

Step 3: Now, DEMATEL and ANP are integrated to assign weights to different SCRE capabilities.

Step 3.1: First, we calculate the values of different criteria with respect to offshore outsourcing benefits. This step is processed by the AHP method using the 5-level scale. The following results are obtained:

Technical aspects: 0.41, Economical aspects: 0.18, Social aspects: 0.18, Environmental aspects: 0.11 , and Political aspects: 0.12

Step 3.2: The initial unweighted supermatrix of ANP can be acquired by entering the vectors derived from DEMATEL and Step 2. The results are shown in the Appendix.

Multiplying the unweighted supermatrix by the criteria relative weights from Step 3.1 produces the normalized supermatrix. The supermatrix is then increased to a sufficiently large power until convergence occurs to obtain the limited supermatrix.

Step 4: The relative weights of the SCRE capabilities are determined with respect to benefits. The final priority values are found in the corresponding columns of the limited supermatrix. The results are shown in the Appendix.

\subsection{Rank alternative locations and select optimal one in SCRE perspective}

In this section, the optimal alternative locations for offshore outsourcing are selected by using TOPSIS based on the existing experts' evaluations. First, a group discussion is conducted in which the experts evaluate 4 potential alternative locations: L1, L2, L3, and L4. In this way, scores are acquired that represent how a location fulfills outer factors. A five-point scale is developed in the Appendix.

The scores of each outer factor for the locations consist of an initial vector, which is then input into the FCM to determine the adjacency as in Section 4.1. After several iterations, the FCM presents an equilibrium state that reflects the level of SCRE capabilities. The concrete process of the evolutionary process is shown in Appendix. In this study, the equilibrium state emerges after more than 4800 iterations. The equilibrium values generally reflect the final SCRE capability: the higher the value, the more elastic the supply chain will be. The sign $(+,-)$ indicates that the supply chain resilience will be enforced or damaged. For example, capability 8 (market position) reaches -0.6990 in location 1 , which indicates that Cap8 will be impaired in location 1 with a degree of 0.699. Cap3 (anticipation) is 0.4296 in location 3 after many iterations. Therefore, Cap3 will be activated to some extent in location 3. The detailed results 
are shown in the Appendix. In this way, the mechanism of evolutionary processes is revealed, and the SCRE capabilities in each location are presented for further decision-making.

Once the equilibrium values of the different capabilities in the 4 locations are obtained, the TOPSIS method is utilized to analyze the consistency of each alternative location with respect to the ideal solution from the resilience perspective. The weights of each capability from Section 4.2 will be considered.

First, we construct a decision matrix $D=\left(x_{m m}\right)$ from Table 13. Then, we normalize the decision matrix by Eq. (12). The weights of each capability are considered in this step, and using Eqs. (14)-(18), the results are calculated and listed in Appendix. The most suitable location considering supply chain resilience is location 3 , whose value is 0.6866 . The different values of the SCRE capabilities in the four locations are shown in Fig. 2.

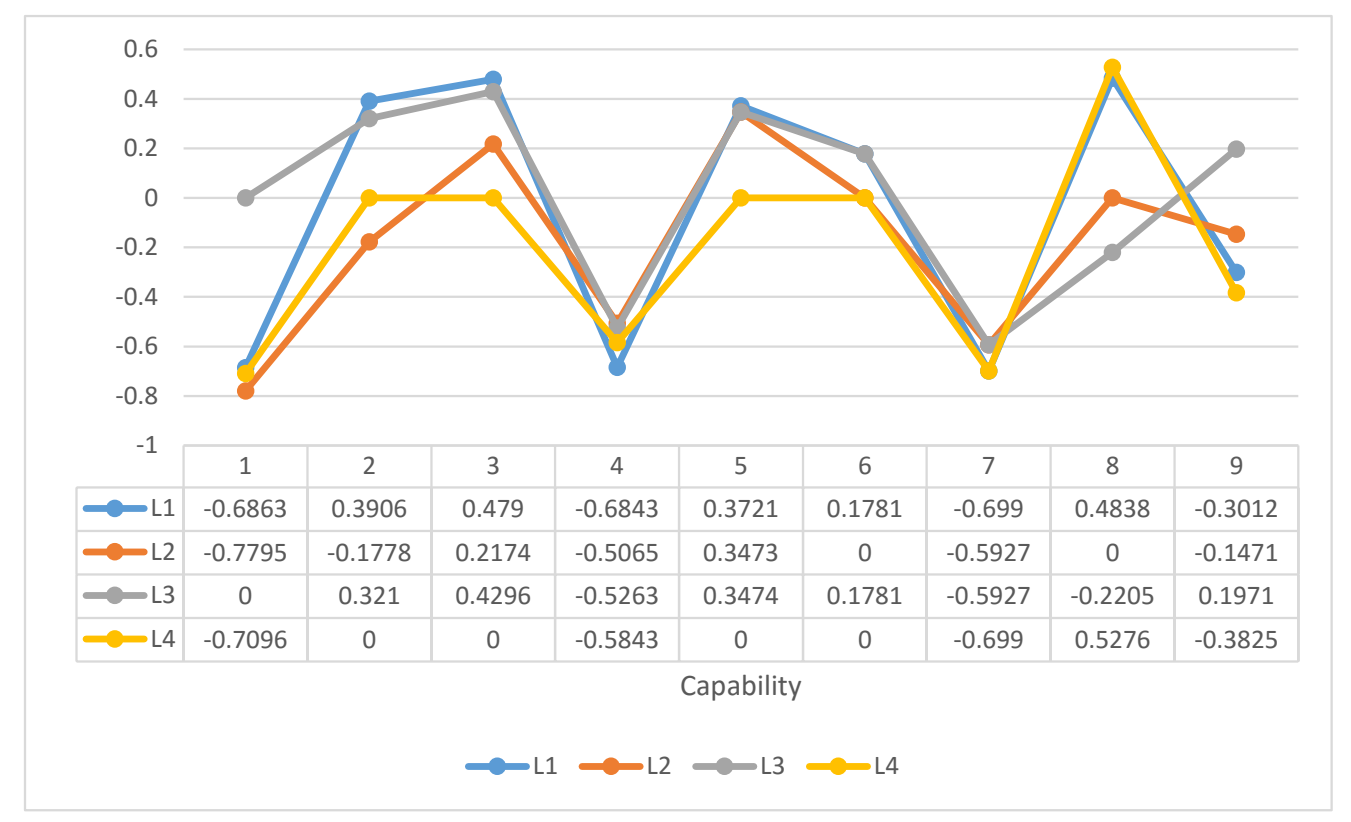

Figure 2: Comparison of the 4 locations

\section{$5 \quad$ Sensitivity analysis}

To investigate the impact of the SCRE's weights (developed by $W_{C a p i}$ for capability Capi, where $i=1,2, \cdots, 9)$ on the selection of offshore outsourcing locations with the best benefits, we conduct a sensitivity analysis. Eleven experiments were conducted. In the Appendix can bee see the details of these experiments.

As shown in the Appendix, in the first nine experiments, the weight of each SCRE capability is set as the highest one by one, and the others are set at low and equal values. For example, in experiment 1, the weight of Cap1 is 0.5 , and the weights of the remaining SCRE capabilities (Cap2-Cap9) are 0.0625. The highest weight is computed as 0.5 as follows. Since there are a total of 9 capabilities, 8 are assumed to be of equal importance and are allocated equal weights of 0.0625 . This leaves the weight of Cap1 as 0.5 , which ensures that the sum of all weights of the capabilities is 1 . Similarly, in the next 8 experiments, we set each capability as the most important one by giving it a weight of 0.5 and leaving equal weights of 0.0625 for the remaining 
capabilities. In experiment 10, the weights of each SCRE capability are equal to 0.111. In experiment 11, the real case in our study is shown.

As shown in the Appendix, location 4 has the highest score in $7 / 11$ experiments (including the case study). Therefore, based on the assessments obtained, our offshore outsourcing location is comparatively insensitive to weights, with location 4 emerging as the winner in the majority of cases $(7 / 11)$. However, in this study, the best option for practitioners is L3. This could be regarded as a special case that reveals that the selection in the real situation may be different than that in the theoretical analysis.

\section{Results of the simulation of four alternative locations}

In this section, we further investigate the acquired results and discuss some implications of the analyzed data. The supply chain resilience values are presented in Table 13. The results are gathered from the FCM dynamic process, which reveals the future trends of supply chain resilience. Although the optimal solution is identified by comparing its distance to the ideal solution, we explore some special cases by using the results of our proposed model.

\subsection{Results of simulation of location 1}

The results show that 5 resilience capabilities will be enforced and 4 resilience capabilities will be damaged. The final values range from -0.6990 to 0.4838 . Visibility (Cap2), anticipation (Cap3) and adaptability (Cap6) are the highest in this location among the four alternatives, which indicates that these three capabilities will be increased to different extents. However, recovery capability (Cap4) and financial strength (Cap7) are significantly impaired compared with the other three alternatives.

\subsection{Results of simulation of location 2}

In location 2, the supply chain's adaptability (Cap4) and market position (Cap8) will not be influenced and thus will remain at a stable level. The anticipation capability (Cap3) and security (Cap5) will increase slightly $(+0.2174$ and +0.3473 , respectively) in location 2 . In addition, other capabilities will be damaged to different extends extents. Among these damaged capabilities, flexibility (Cap1) is greatly impaired, even compared with other places. Companies seeking flexibility in their supply chain should avoid choosing this location.

\subsection{Results of simulation of location 3}

In location 3 , the flexibility of the supply chain will be stable and will not be influenced. Five capabilities (Cap2, Cap3, Cap5, Cap6, and Cap9) will be improved. Among those capabilities, adaptability (Cap6) and collaboration (Cap9) have their highest values at this location among the four alternatives, whereas the market position of this location has the lowest value compared with the other alternatives. Companies that are concerned with adaptability and collaboration should pay more attention to this location.

\subsection{Results of simulation of location 4}

The supply chain's capabilities of visibility (Cap2), anticipation (Cap3), security (Cap5), and adaptability (Cap6) are not influenced in location. It has a comparatively good market position among the four alternatives (the value is 0.5276). However, other resilience capabilities will be impaired. 
Since offshore outsourcing refers to the transfer of some specific ownership business activities or resources to low-cost providers outside of the client company's country of origin, a new provider will be a new player in the SC network. The offshore outsourcing process can exert some impacts on SC resilience compared to in-home production. Many capabilities of SC resilience are enhanced, while some capabilities are weakened. In this part, we discuss the differences between offshore outsourcing and in-home production with respect to $\mathrm{SC}$ resilience. Hence, if a manager seeks to preserve or even improve some capabilities of SC resilience, they should consider whether or not to select offshore outsourcing; if they choose offshore outsourcing, they should also consider which location to select.

\section{Conclusions and future works}

Offshore outsourcing is a heated issue that has received extensive attention. However, the impacts of complicated and diverse environments on supply chain resilience may minimize the ability of supply chains to defend against risks. To overcome this problem, we propose to select a suitable location where the supply chain's resilience will be maximized based on an integrated MCDM method. The main contributions and innovations of this research paper could be summarized as follows. Firstly, this paper combines FCM and D number theory to the concept development if DCM that not only remains the abilities to represent uncertainty but also contributes to aggregating knowledge from different sources (experts/commanders). Since uncertain information fusion has been studied for many years, indicating that D number theory is an effective framework to represent and fuse uncertain information. The combination of D number theory and FCM is shown to be valuable approaches through illustration. Secondly, DEMATELANP is implemented to quantify the weight in context of offshore outsourcing problem. From sensitivity analysis, we conclude that a concise result could be obtained. Overall, The proposed method can support practitioners while evaluating alternative outsourcing locations according to their impacts on the SC resilience. Experts perceived the main advantage of the proposed method in the ability to predict effects due to indirect implications, which are otherwise very difficult to predict, especially for large models. In fact, in our case, one location would improve resilient capabilities and two locations would rather damage it. Such location behavior could not been predicted without our simulation. For academics, this paper provides a groundwork for further studies because it is the first time that a research shows how offshore outsourcing location decision-making can improve, preserve or damage SC resilience. Looking to the future, empirical works would validate the influences detected. In addition, the developed hybrid method is generic, flexible and easily adaptable. Therefore, it could be applied easily to other sectors to represent messy problems with causalities and predict future outcomes.

Our future work involves the validation of the proposed model results by comparison with other techniques available in the literature for the selection of outsourcing locations.

\section{Funding}

This research is supported by the Fundamental Research Funds for the Central Universities(No. XDJK2019C085) and Chongqing Overseas Scholars Innovation Program(No. cx2018077).

\section{Author contributions}

L.Z designed, performed experiments and wrote the paper. F.X developed the method, conceived the experiments and revised the paper. 


\section{Conflict of interests}

The authors declare no conflict of interest.

\section{Bibliography}

[1] Ali, S.; Hongqi, L.; Khan, S.U.; Zhongguo, Y.; Liping, Z. (2017. Success factors for software outsourcing partnership management: An exploratory study using systematic literature review. IEEE Access, 5, 23589-23612, 2017.

[2] Dekkers, R.(2000). Decision models for outsourcing and cor competencies in manufacturing. International Journal of Production Research, 38(17), 4085-4096, 2000.

[3] Dempster, A.P. (1967). Upper and lower probabilities induced by a multivalued mapping. The annals of mathematical statistics, 325-339, 1967.

[4] Dong, Y.; Zhang, J.; Li, Z.; Hu, Y.; Deng, Y.(2019). Combination of evidential sensor reports with distance function and belief entropy in fault diagnosis. International Journal of Computers Communications \& Control, 14(3), 293-307, 2019.

[5] Fontela, E.; Gabus, A.(1976). The dematel observer, Battelle Geneva Research Center, Geneva, 1976.

[6] Gao, X.; Deng, Y. (2019). The generalization negation of probability distribution and its application in target recognition based on sensor fusion. International Journal of Distributed Sensor Networks,15(5), 381, 2019.

[7] Giret, A.; Julián, V.; Corchado, J.M.; Fernández, A.; Salido, M.A.; Tang, D.(2018). How to choose the greenest delivery plan: A framework to measure key performance indicators for sustainable urban logistics. IFIP International Conference on Advances in Production Management Systems, 181-189, 2018.

[8] Guan, X.; Liu, H.; Yi, X.; Zhao, J.(2018). The Improved Combination Rule of D Numbers and Its Application in Radiation Source Identification, Mathematical Problems in Engeneering, Article ID 6025680, 2018.

[9] Gurtu, A.; Searcy, C.; Jaber, M. (2016). Effects of offshore outsourcing on a nation. Sustainable Production and Consumption, 7, 94-105, 2016.

[10] Gylling, M.; Heikkilä, J.; Jussila, K.; Saarinen, M. (2015). Making decisions on offshore outsourcing and backshoring: A case study in the bicycle industry. International Journal of Production Economics, 162, 92-100, 2015.

[11] Han, Y.; Deng, Y.(2018). An enhanced fuzzy evidential dematel method with its application to identify critical success factors. Soft computing, 22(15), 5073-5090, 2018.

[12] Han, Y.; Deng, Y. (2018). An evidential fractal ahp target recognition method. Defence Science Journal, 68(4), 367-373, 2018.

[13] Han, Y.; Deng, Y.(2018). A hybrid intelligent model for assessment of critical Ambient intelligence, 9(6), 1933-1953, 2018.

[14] Han, Y.; Deng, Y. (2019). A novel matrix game with payoffs of maxitive belief structure, International Journal of Intelligent Systems, 34(4), 690-706 
[15] Han, Y.; Deng, Y.; Cao, Z.; Lin, C.(2019). An interval-valued pythagorean prioritized operator-based game theoretical framework with its applications in multicriteria group decision making, Neural Computing and Applications, 1-19, 2019.

[16] Lai, Y.J.; Liu, T.Y.; Hwang, C.L.(1994). Topsis for modm. European journal of operational research, 76(3), 486-500, 1994.

[17] Li, Y.; Deng, Y.(2019). TDBF: Two Dimension Belief Function. International Journal of Intelligent Systems, 34(8), 1968-1982, 2019.

[18] Lin, S.; Li, C.; Xu, F.; Liu, D.; Liu, J. (2018). Risk identification and analysis for new energy power system in China based on D numbers and decision-making trial and evaluation laboratory (DEMATEL), Journal of Cleaner Production,180, 81-96, 2018.

[19] Liu, P.; Zhang, X.(2019). A multicriteria decision-making approach with linguistic D numbers based on the Choquet integral, Cognitive Computation, 11(4), 560-575, 2019.

[20] Mo, H.; Deng, Y.(2019). An evaluation for sustainable mobility extended by D numbers, Technological and Economic Development of Economy, 25(5), 802-819, 2019.

[21] Mousavi, S.M.; Antuchevičienè, J.; Zavadskas, E.K.; Vahdani, B.; Hashemi, H.(2019). A new decision model for cross-docking center location in logistics networks under interval-valued intuitionistic fuzzy uncertainty, Transport, 34(1), 30-40, 2019.

[22] Mukherjee, D.; Gaur, A.S.; Datta, A.(2013). Creating value through offshore outsourcing: An integrative framework, Journal of International Management, 19(4), 377-389, 2013.

[23] O'keefe, J.; Nadel, L.(1978). The hippocampus as a cognitive map, Clarendon Press, Oxford, 1978.

[24] Papageorgiou, E.; Stylios, C.; Groumpos, P.(2003). Fuzzy cognitive map learning based on nonlinear hebbian rule, Australasian Joint Conference on Artificial Intelligence, 256-268, 2003.

[25] Pereira, V.; Anderson, V.(2012). A longitudinal examination of hrm in a human resources offshoring (hro) organization operating from india. Journal of World Business, 47(2), 223$231,2012$.

[26] Saaty, T.L.(1980). The analytic hierarchy process: planning. Priority Setting. Resource Allocation, MacGraw-Hill, New York International Book Company, 1980.

[27] Saaty, T.L.(1996). Decision making with dependence and feedback. The analytic network process, Rws Publications, 1996.

[28] Shafer, G., et al.(1976). A mathematical theory of evidence, vol. 1. Princeton university press, Princeton, 1976.

[29] Shankar, R.; Choudhary, D.; Jharkharia, S.(2018). An integrated risk assessment model: A case of sustainable freight transportation systems, Transportation Research Part D: Transport and Environment, 63, 662-676, 2018.

[30] Shukla, A.; Agarwal, P.; Rana, R.; Purohit, R.(2017). Applications of topsis algorithm on various manufacturing processes: A review. Materials Today: Proceedings, 4(4), 5320-5329, 2017. 
[31] Singh, P.; Agrawal, R.(2018). A customer centric best connected channel model for heterogeneous and iot networks, Journal of Organizational and End User Computing, 30(4), $32-50,2018$.

[32] Sun, R.; Deng, Y.(2019). A new method to identify incomplete frame of discernment in evidence theory, IEEE Access, 7, 15547-15555

[33] Sun, R.; Deng, Y.(2019). A new method to determine generalized basic probability assignment in the open world, IEEE Access, 7, 52827-52835, 2019.

[34] Tsai, S.B.; Zhou, J.; Gao, Y.; Wang, J.; Li, G.; Zheng, Y.; Ren, P.; Xu, W.(2017). Combining fmea with dematel models to solve production process problems. Plos One, 12(8), e0183, $634,2017$.

[35] Yadlapalli, A.; Rahman, S.; Gunasekaran, A.(2018). Socially responsible governance mechanisms for manufacturing firms in apparel supply chains. International Journal of Production Economics, 196, 135-149, 2018.

[36] Yang, H.; Deng, Y. (2019). A bio-inspired optimal network division method, Physica A: Statistical Mechanics \& Its Applications, 527, 210-219

[37] Yang, H.; Deng, Y.; Jones, J. (2018). Network division method based on cellular growth and physarum-inspired network adaptation, International Journal of Unconventional Computing, 13(6), 477-491, 2018.

[38] Yoon, K.; Hwang, C.L. (1981). Multiple attribute decision making: methods and applications, Springer-Verlag, Berlin, 1981.

[39] Yoon, K.P.; Kim, W.K.(2017). The behavioral topsis. Expert Systems with Applications, 89, 266-272, 2017. 